\title{
Spontaneous orbiting of two spheres levitated in a vibrated liquid
}

\author{
H. A. Pacheco-Martinez, L. Liao, R. J. A. Hill, Michael R. Swift and R. M. Bowley \\ School of Physics and Astronomy, \\ University of Nottingham, \\ Nottingham, NG7 2RD, U.K.
}

\begin{abstract}
In the absence of gravity, particles can form a suspension in a liquid irrespective of the difference in density between the solid and the liquid. If such a suspension is subjected to vibration there is relative motion between the particles and the fluid which can lead to self-organization and pattern formation. Here we describe experiments carried out to investigate the behavior of two identical spheres suspended magnetically in a fluid, mimicking weightless conditions. Under vibration the spheres mutually attract and, for sufficiently large vibration amplitudes, the spheres are observed to spontaneously orbit each other. The collapse of the experimental data onto a single curve indicates that the instability occurs at a critical value of the streaming Reynolds number. Simulations reproduce the observed behaviour qualitatively and quantitatively, and are used to identify the features of the flow that are responsible for this instability.
\end{abstract}

PACS numbers: 47.32.-y, 47.57.E-, 85.70.Rp, 47.20.Ky

In the absence of gravity any collection of particles can form a suspension in a liquid, regardless of their density. If the densities are different and the suspension is vibrated, the particles and the fluid will move relative to each other. The resulting hydrodynamic flows in the liquid can strongly influence the behavior of the suspended particles. Here we show how diamagnetic levitation can be used to study such a suspension, reproducing the effect of weightless conditions in an orbiting spacecraft. We study the behavior of two spheres suspended in a liquid that is vibrated to induce hydrodynamic flows around the spheres.

Hydrodynamic interactions between particles are a known mechanism for inducing dynamic self-assembly [1]. Ordinarily, such experiments are limited to twodimensional ordering due to the presence of gravity, either with the particles on a surface [2-6], confined to the interface between two immiscible liquids $[7,8]$, or suspended in the liquid via a rod [9-12]. In a zero-gravity environment, such as on the International Space Station, three-dimensional suspensions of particles have been used in crystal growth $[13,14]$. It is found that stray vibrations (g-jitter) [15] generate undesirable hydrodynamic flows around the growing crystallites, which are known to reduce the quality of the resulting crystals [16].

A strong magnetic field with a large vertical field gradient can be used to suspend particles in a liquid, even if the density of the particles is much greater than that of the liquid [17]. Such an arrangement allows us to investigate non-linear hydrodynamic effects in a fully threedimensional fluid-particle system.

In this Letter we report experiments and simulations carried out to investigate a novel hydrodynamic instability exhibited by two identical spheres suspended magnetically and vibrated freely in a liquid. Under vertical vibration the spheres are attracted to each other and align so that the line joining their centers lies perpendicular to the axis of vibration [2-5]. As the amplitude of vi- bration is increased beyond a critical value, the spheres orbit each other in the horizontal plane, with no preferred sense of rotation. We have investigated the conditions for the onset of this instability in terms of the viscosity of the liquid, the amplitude and frequency of the oscillation and the size and density of the spheres. We have also carried out simulations which reproduce the behavior observed experimentally both qualitatively and quantitatively. The experiments and simulations reveal the existence of two jets emerging from the point of contact of the two spheres. Above a critical amplitude of vibration, the angle of the jets changes giving rise to a net torque on the spheres.

Our experiments were carried out using a 17 Tesla superconducting magnet (Oxford Instruments), details of which are given in ref. [18]. Conditions for suspension are given in the supplementary information [19], see also [17]. Pairs of equal-sized, highly spherical glass ball lenses (CVI Melles Griot) of diameters $d=1,2$ or 3 $\mathrm{mm}$ were placed inside a transparent cylindrical cell of internal diameter $34 \mathrm{~mm}$ and height $110 \mathrm{~mm}$, containing a paramagnetic solution of $\mathrm{MnCl}_{2}$. Varying amounts of glycerol were added to adjust the fluid's viscosity. The cell was inserted into the vertical bore of the magnet, and connected by a drive rod to a loudspeaker mounted beneath the magnet [18] (supplementary fig. S1 [19]). The spheres "levitate" in stable mechanical equilibrium at a local minimum in the magneto-gravitational potential energy [20-22]. We measured the dimensionless peak acceleration $\Gamma=A \omega^{2} / g$ (where $\omega=2 \pi f$ is the angular frequency of vibration) using an accelerometer mounted on the loudspeaker, from which we determine the amplitude of vibration, $A$. Typically $\Gamma$ was less than 7 , the frequency $f$ was in the range $12-35 \mathrm{~Hz}$, and the amplitude was less than $5 \mathrm{~mm}$. The cell was positioned so that the stable levitation point was located near the cell's center.

Experiments were also carried out using a pair of bismuth spheres which could be suspended in a water- 


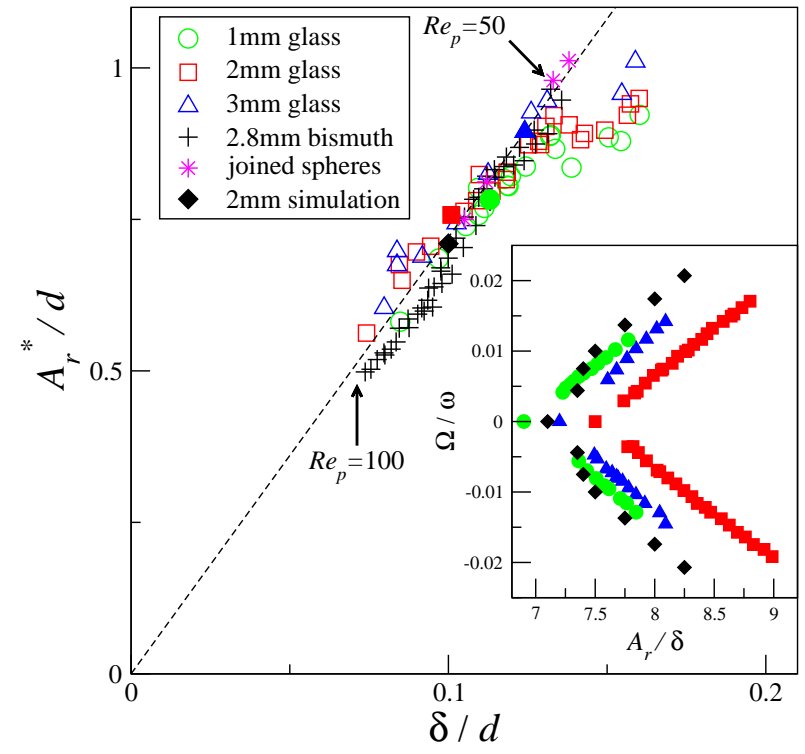

FIG. 1: The main panel shows the collapse of the onset data in terms of the dimensionless variables $A_{r}^{*} / d$ and $\delta / d$. The kinematic viscosity is in the range $1.13-2.43 \mathrm{~mm}^{2} \mathrm{~s}^{-1}$ for 1 $\mathrm{mm}$ glass spheres; $2.76-8.37 \mathrm{~mm}^{2} \mathrm{~s}^{-1}, 2 \mathrm{~mm}$ glass; $7.14-$ $14.9 \mathrm{~mm}^{2} \mathrm{~s}^{-1}, 3 \mathrm{~mm}$ glass; and $6.46-15.6 \mathrm{~mm}^{2} \mathrm{~s}^{-1}, 2.8 \mathrm{~mm}$ bismuth shot. The inset shows the dependence of the scaled orbital angular velocity $\Omega / \omega$ on the scaled relative amplitude $A_{r} / \delta$ for representative data sets. The points at $\Omega / \omega=0$ are extrapolations; onsets determined from these points are shown by filled symbols on the main panel.

glycerol mixture. In some of these experiments finely crushed bismuth was added to the fluid. In the magnetic field the bismuth powder levitated in the same region as the pair of bismuth spheres. Under vibration the powder formed a halo around the spheres which, owing to the high reflectivity of the metal grains, could be used to image the flow of the liquid in the vicinity of the spheres [19].

Under vertical vibration, the spheres move relative to the fluid owing to the difference between the inertial mass of the particles and the fluid they displace, but do not sediment owing to the equality of their effective gravitational masses: the gravitational force on the spheres is balanced by a buoyancy force that is enhanced by a magnetic force on the fluid. The spheres attract each other due to the presence of streaming flows $[23,24]$ that form when the cell is vibrated; these flows align the spheres horizontally with the line joining their centers perpendicular to the direction of vibration $[2,3]$.

We first describe the behavior of the glass spheres in $\mathrm{MnCl}_{2}$ solution as we increased the amplitude $A$ for a fixed $\omega$. For low values of $A$ the spheres moved vertically in response to the fluid motion, but there was no motion of the spheres in the horizontal plane. Above a critical value of $A$, which we label $A^{*}$, the spheres orbited each other at a constant angular speed in the horizontal plane.

Although $A$ is the quantity we measure experimentally,

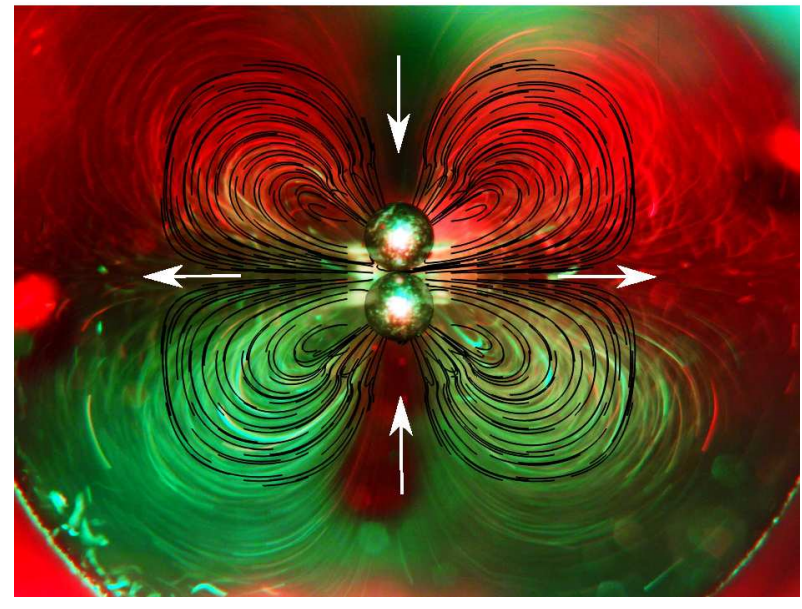

FIG. 2: An image of the spheres taken from above when the fluid is vibrated but there is no orbital motion. Colour is used to enhance contrast. The vibration is applied along the direction perpendicular to the plane of this image. The red and green streak lines indicate the steady streaming flow in the plane of the spheres. There is a strong outward flow from the contact point of the spheres and an inward flow elsewhere, as illustrated by the white arrows. The superimposed black lines show the corresponding streak lines obtained from simulation.

the relevant amplitude characterising the behaviour is that of the oscillatory motion of the spheres relative to the liquid far from the spheres, $A_{r}$. In general the relative motion of the spheres differs in amplitude and phase from the motion of the cell [25]. We determined $A_{r}$ of the pair of spheres as a function of the driving amplitude $A$, angular frequency $\omega$, and kinematic viscosity $\nu$ in an independent set of experiments.

To quantify the instability we measured the relative amplitude of vibration at the onset of the orbital motion $A_{r}^{*}$ for a range of particle sizes, fluid viscosities and vibration frequencies. For $A_{r}>A_{r}^{*}$ the particles orbited each other. As $A_{r}$ was increased above $A_{r}^{*}$, the particles orbited at a faster rate and sometimes separated for higher relative amplitudes. We obtained $A_{r}^{*}$ by first increasing $A_{r}$ until rotation was observed. We then decreased $A_{r}$ slowly, measuring the rate of rotation $\Omega$ for each value of $A_{r}$. Example data are shown in the inset to Fig. 1. For small $\Omega / \omega$ there is an approximate linear dependence on $A_{r} / \delta$. The onset amplitude $A_{r}^{*}$ is determined by extrapolating the linear portion of the data to zero rotation rate $\Omega=0$.

If the transition is purely hydrodynamic (rather than a magneto-hydrodynamic effect [19] or induced by unwanted vibrations of the apparatus) the onset can only depend on three independent length scales : $A_{r}^{*}, d$, and the viscous penetration depth $\delta=(\nu / \omega)^{1 / 2}$. Here we assume that the cell is sufficiently large as to have no influence on the instability; in all our experiments the ratio of the diameter of the cell to the diameter of the particle is greater than 10 . In Fig. 1 we plot the ratio $A_{r}^{*} / d$ as a function of $\delta / d$ for the parameters given in the 


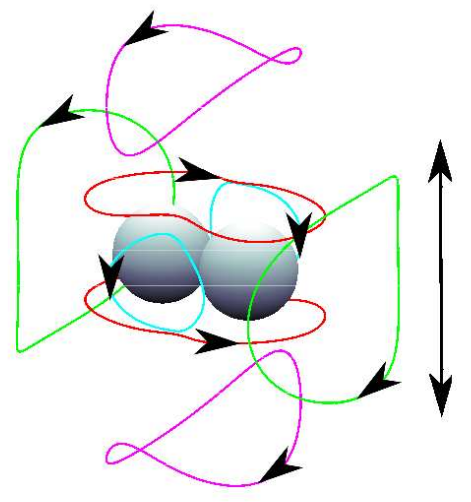

FIG. 3: A representation, obtained from simulation, of the time-averaged vortex structure around the two spheres vibrated under conditions for which they are not rotating. The closed loops represent the curl of the velocity field and are schematic vortex lines as described in the text. The direction of the arrows indicates the sense of rotation i.e. the direction of the curl of the velocity field. The double-headed arrow indicates the direction of vibration.

figure caption. It can be seen that there is a good collapse of the data onto a single curve within experimental error which confirms our assumptions that the transition is purely hydrodynamic and that the cell is sufficiently large. The part of the curve for $\delta / d<0.13$ (corresponding to a particle Reynolds number $R e_{p}=A_{r} d / \delta^{2} \gtrsim 50$ ) lies close to a straight line through the origin, shown in Fig. 1. This line corresponds to a value of the streaming Reynolds number $R e_{s}=A_{r}^{2} / \delta^{2} \simeq 52$, which is independent of the particle size [23].

For low values of $A_{r} / d$ and $\delta / d$ there is no consistent rotational instability. The lower limit of $\delta / d$ for which we observe rotation corresponds to an upper limit on the particle Reynolds number $R e_{p} \simeq 100$ as indicated by the lower arrow in Fig.1. For higher values of $R e_{p}$ the particle's motion becomes unsteady and no periodic orbital motion is observed. We speculate that this behavior might be due to vortex shedding as has been observed in simulation for a single sphere [26].

For $\delta / d$ greater than about $0.13\left(R e_{p} \lesssim 50\right)$ a gap opens up between the particles before the spheres start to orbit [3]. The data for which there is a gap appears to collapse onto the hook-shaped part of the curve. However, if the spheres are stuck together with glue, the onset of orbital motion falls on to the straight line through the origin, as shown in Fig. 1. Our data is bounded for larger values of $\delta / d$ by the limits of our experimental setup: the vibratory waveform becomes significantly non-sinusoidal below about $11 \mathrm{~Hz}$.

The inset to Fig. 1 shows the orbital angular velocity $\Omega$ as a function of the relative amplitude $A_{r}$ for representative sets of data. Rotation rates are symmetric for the clockwise and anticlockwise directions. Markers on the spheres show that during the motion the spheres appear

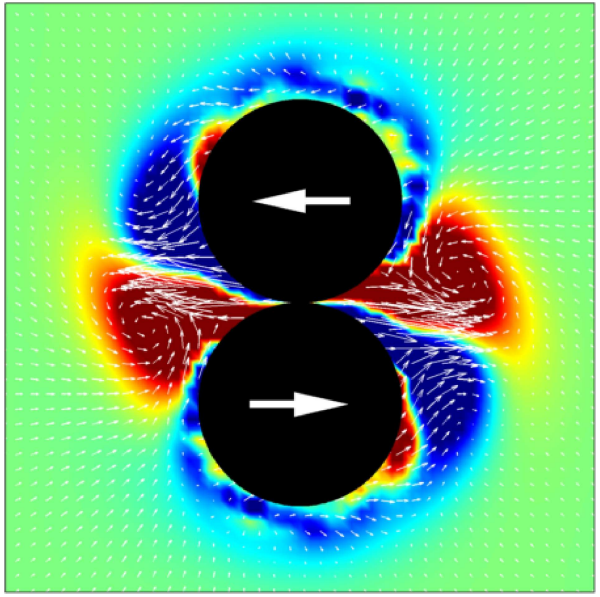

FIG. 4: Data from simulations showing the instantaneous flow field and vorticity map in the equatorial plane of the spheres under conditions for which they orbit anti-clockwise. The direction of vibration is normal to the plane of the figure. The small white arrows indicate the instantaneous flow and the background colour map indicates the strength and sense of the vorticity. Large arrows indicate the direction of the orbital motion.

to slide around each other, with the markers maintaining their orientation with respect to the cell. As $A_{r}$ increases, the orbital angular velocity $\Omega$ increases. Eventually the particles separate at high rotation rates and the orbital motion can become unstable. In general, the rotation data cannot be collapsed in terms of $A_{r} / d$ and $\delta / d$ because the introduction of an orbital time scale gives rise to an extra length scale $l=(\Omega / \nu)^{1 / 2}$. However, the extrapolated value of $A_{r} / \delta$ to zero rotation rate should all be the same, which they are within experimental error arising from uncertainties in the measurement of the viscosity.

Further experiments were carried out using bismuth shot to ascertain the effect of changing the particle density. Using bismuth has the added benefit that we can use crushed bismuth as tracer particles to help visualize the streaming flow. An example of the flow pattern is shown in Fig. 2. In this long-exposure image, the streaklines traced out by the bismuth powder appear as white trails. The streakline photograph is focused to show the horizontal flow in the plane through the centers of the two spheres. The bismuth shot behaved in the same way as the glass spheres, giving a similar data collapse for the onset of orbital motion, as shown in Fig. 1. When orbiting, the two bismuth shot particles move as a single solid object instead of sliding past each other as the glass spheres do. We attribute this behavior to the roughness of the bismuth surface. However, this difference in motion is irrelevant as far as the onset of orbital motion is concerned.

We were unable to obtain good images of the full threedimensional flow because of the confined space in the bore of the magnet. In order to more fully understand 
the streaming flows and the cause of the instability we carried out simulations based on a molecular-dynamics treatment of the particles coupled to a numerical solution of the continuum Navier-Stokes equations for the fluid. Details of the simulation method and its validation can be found in references $[3,5,27]$ and in the supplementary information [19].

Some data from simulation showing the rotation rates and extrapolated onset amplitude are shown in the inset and main panel of Fig. 1. The simulated streaming flows under conditions for which there is no orbital motion, i.e. $A_{r}<A_{r}^{*}$, are shown as black lines superposed onto the photograph in Fig. 2. The simulation parameters were similar to those in the experiments. To reduce numerical noise we simulated vibration of the particles with the appropriate $A_{r}$ in a static fluid. It can be seen from Figs. 1 and 2 that the simulated rotation rates, onset amplitude $A_{r}^{*}$ and flows are in good quantitative agreement with those found experimentally. The difference between the simulated flow and experimentally observed flow at large distances $(\gtrsim 5 d)$ is due to finite size effects in the simulation.

In both experiment and simulation we observe that, in the plane of the two spheres, there are two strong outward-flowing jets emerging from the region between the two spheres and an inward flow elsewhere, as illustrated by the arrows on Fig. 2. This behavior is completely different from that of a single sphere under equivalent conditions, where the equatorial flow is radially inwards towards the sphere everywhere [24, 26].

Using the simulations, we investigated the structure of the full three-dimensional flow around the two spheres. The local flow is best visualized from the vorticity of the time-averaged velocity of the fluid. Fig. 3 shows four pairs of loops; the arrows on the loops indicate the direction of the circulation (local curl of the velocity field) from which the fluid flow can be obtained by the righthand screw rule. The four pairs of loops represent all the basic features of the flow pattern around the spheres, but do not indicate its magnitude. The upper and lower loops (pink) on the diagram show that, above and below the spheres, the flow is predominantly away from the spheres (i.e. up and down), as is the case for a single sphere or two spheres vibrated on rigid rods [11]. The left and right outer loops (green) represent inward flow towards the spheres; for a single sphere this flow would also be present but in that case the equatorial flow is inward everywhere. The two, small inner loops (blue) represent the strong horizontal outward flow from between the spheres, something which is not present for a single sphere. The inner loops immediately above and below the spheres (red) feed this outward flow.

As the amplitude is increased the vortices associated with the jets become larger and the mirror symmetry of the equatorial flow pattern, evident in Figs.2 and 3, is broken. Now the direction of the jet is no longer perpendicular to the line through the center of the two spheres, as shown in Fig. 4. It is this broken symmetry that gives rise to a net torque on the two spheres causing the orbital motion. The steady rotation rates that we observe, shown in the inset to Fig. 1, are governed by the balance between this torque and the viscous drag in the fluid.

Our findings show the usefulness of magnetic levitation to investigate a fundamental problem in hydrodynamics. This technique has allowed us to explore the full threedimensional flow around vibrated particulates without the complications of boundary effects. We anticipate that our work will open up new ways for manipulating and ordering granular suspensions in a non-invasive way.

Acknowledgements: This work was supported by a Basic Technology Grant from EPSRC, UK; grant nos. GR/S83005/01 and EP/G037647/1. R.J.A.H. acknowledges EPSRC for support under a Research Fellowship; grant no. EP/I004599/1. We are grateful to D. Klotsa for many helpful discussions.
[1] G. M. Whitesides and B. Grzybowski, Science, 295, 2418 (2002).

[2] G. A. Voth, B. Bigger, M. R. Buckley, W. Losert, M. P. Brenner, H. A. Stone and J. P. Gollub, Phys. Rev. Lett, 28, 234301 (2002).

[3] D. Klotsa, M. R. Swift, R. M. Bowley and P. J. King, Phys. Rev. E, 76, 056314 (2007).

[4] R. Wunenburger, V. Carrier and Y. Garrabos, Physics of Fluids, 14, 2350 (2002).

[5] D. Klotsa, M. R. Swift, R. M. Bowley and P. J. King, Phys. Rev. E, 79, 021302 (2009).

[6] H. S. Wright, M. R. Swift and P. J. King, Phys. Rev. E, 78, 036311 (2008)

[7] A. Snezhko and I. S. Aranson, Nature Materials, 10, 698 (2011).

[8] M. Belkin, A. Snezhko, I. S. Aranson I. S. and W. K. Kwok, Phys. Rev. Lett., 99, 158301 (2007).

[9] S. Hassan, T. P. Lyubimova, D. V. Lyubimov and M.
Kawaji, Trans. A.S.M.E., 73, 72 (2006).

[10] S. Hassan, T. P. Lyubimova, D. V. Lyubimov and M. Kawaji, Int. J. of Multiphase Flow, 32, 1037 (2006).

[11] F. Otto, E. K. Riegler and G. A. Voth, Physics of Fluids, 20, 093304 (2008).

[12] C. K. Kotas, M. Yoda and P. H. Rogers, Exp. Fluids 42, 111 (2007).

[13] L. DeLucas et al., Science, 246, 651 (4930).

[14] N. E. Chayen, E. H. Snell, J. R. Helliwell and P. F. Zagalsky, J. Crystal Growth, 171, 219 (1997).

[15] W. Knabe and D. Eilers, Acta Astronautica, 9, 187 (1982).

[16] B. Lorber, J. D. Ng, P. Lautenschlager and R. Giegé, J. Crystal Growth, 208, 665 (2000).

[17] A. T. Catherall, P. J. King, L. Eaves and S. R. Booth, Nature 422, 579 (2003); A. T. Catherall, P. López-Alcaraz, K. A. Benedict, P. J. King and L. Eaves, New J. Phys., 7, 118 (2005). 
[18] A. T. Catherall, P. López-Alcaraz, P. Sánchez, M. R. Swift and P. J. King, Phys. Rev. E, 71, 021303 (2005).

[19] See Supplemental Material at [URL] for experimental details and movies showing the behavior of the spheres and fluid under different vibratory conditions.

[20] M. V. Berry, A. K. Geim, Eur. J. Phys., 18, 307 (1997).

[21] J. S. Brooks, J. A. Reavis, R. A. Medwood, T. F. Stalcup, M. W. Miesel, E. Steinberg, L. Arnowitz, C. C. Stover, J. A. A. J. Perenboom, J. Appl. Phys., 87, 6194 (2000).

[22] R. J. A. Hill, L. Eaves, Phys. Rev. E 81, 056312 (2010); ibid. 85, 017301 (2012).
[23] G. K. Batchelor, An Introduction to Fluid Dynamics (Cambridge University Press, Cambridge 1967).

[24] N. Riley, Ann. Rev. Fluid Mech., 33, 43 (2001).

[25] C. F. M. Coimbra, D. L'Esperance, R. A. Lambert, J. D. Trolinger and R. H. Rangel, J. Fluid Mech., 504, 353 (2004).

[26] H. M. Blackburn, Phys. of Fluids, 14, 3997 (2002).

[27] K. D. Klotsa, PhD Thesis, University of Nottingham (2009). 